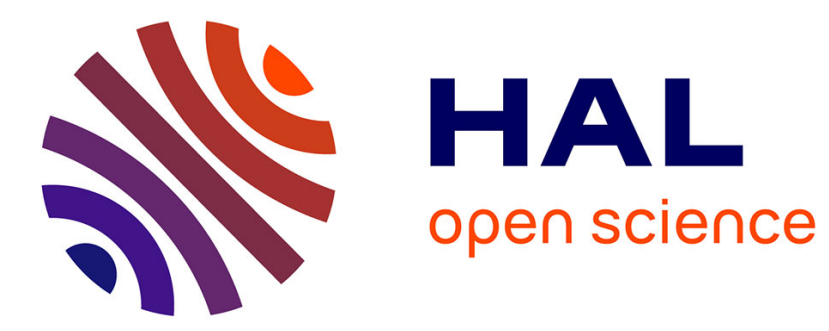

\title{
Helium Atom Beam Scattering as a Probe for the Kinetics on Singular and Vicinal Surfaces: Fundamental Aspects of Ordering, Growth and Ablation H.-J. Ernst
}

\section{- To cite this version:}

H.-J. Ernst. Helium Atom Beam Scattering as a Probe for the Kinetics on Singular and Vicinal Surfaces: Fundamental Aspects of Ordering, Growth and Ablation. Journal de Physique IV Proceedings, 1997, 07 (C6), pp.C6-55-C6-64. 10.1051/jp4:1997605 . jpa-00255704

HAL Id: jpa-00255704

https://hal.science/jpa-00255704

Submitted on 1 Jan 1997

HAL is a multi-disciplinary open access archive for the deposit and dissemination of scientific research documents, whether they are published or not. The documents may come from teaching and research institutions in France or abroad, or from public or private research centers.
L'archive ouverte pluridisciplinaire HAL, est destinée au dépôt et à la diffusion de documents scientifiques de niveau recherche, publiés ou non, émanant des établissements d'enseignement et de recherche français ou étrangers, des laboratoires publics ou privés. 


\title{
Helium Atom Beam Scattering as a Probe for the Kinetics on Singular and Vicinal Surfaces: Fundamental Aspects of Ordering, Growth and Ablation
}

\author{
H.-J. Ernst \\ CEA Saclay, DSM/DRECAM/SRSIM, 91191 Gif-sur-Yvette, France
}

\begin{abstract}
Helium Atom Beam Scattering (HAS) allows for a detailed structural characterization of growth and ablation processes in situ, on an angstrom scale, and in real time. Its application to the growth, ablation and ordering of thin $\mathrm{Cu}$ films on singular and vicinal $\mathrm{Cu}$ templates is briefly reviewed. The investigation of such homoepitaxial systems allows to address the kinetic aspect of growth and ablation in its pure form. It appears this way that in both processes an asymmetry in the horizontal and vertical mobility of atoms, associated with the presence of an excess energy barrier to diffusion at step edges, plays a key role in the formation of the observed selforganized patterned structures on the nanometerscale.
\end{abstract}

\section{INTRODUCTION}

Molecular Beam Epitaxy (MBE) is one of the techniques frequently used for the growth of technologically relevant thin films and multilayer structures. However, while the elementary processes involved are conceptually simple (adsorption, diffusion, and incorporation of adatoms into the growing film), the resulting structure and morphology can not yet be reliably predicted. One of the reasons for this lack of prediction can certainly be attributed to the difficulties in handling non-equilibrium phenomena with many different energetic and kinetic processes competing. Moreover, the resulting thin film morphology is not necessarily determined by the free energy of the system, but may look very different because of kinetic limitations. An elegant way [1,2] to elucidate this aspect of thin film growth and, at the same time, to minimize the number of relevant parameters is to study the growth of a film on a substrate of the the same nature: from a thermodynamic point of view, the growth of crystals in such so called homoepitaxial systems should be structurally perfect (when nonreconstructed and below their roughening temperature), and in the ideal case, proceed in stacking "atomic layer by atomic layer". If this is not observed, one can be certain that the origin has to be a kinetic limitation, because in these systems the surface morphology is exclusively determined by the competition between the roughness induced by the stochastic nature of the impinging atomic flux and the ordering kinetics of adatoms. Thus, homoepitaxial systems allow for a detailed investigation of kinetic processes in their pure form, without the influence of geometrical or chemical mismatches, otherwise almost always present. To this end; we have investigated the growth, ablation and ordering of $\mathrm{Cu}$ of singular and vicinal $\mathrm{Cu}$ templates.

\section{EXPERIMENTAL}

The experiments are performed with the Helium Atom Beam Scattering (HAS) spectrometer "Ramses II" at Saclay. In brief, a supersonic, monoenergetic nozzle beam of He atoms is generated by expanding helium gas at a stagnation pressure of about 150 bar through a nozzel of nominally $10 \mu \mathrm{m}$ in diameter. In this process, random kinetic energy is converted in the course of many collisions into forward kinetic energy. The final velocity distribution of atoms (and therefore the de Broglie wavelength distribution) in the beam is thus sharply peaked $(\Delta v / v \approx 1 \%)$, in contrast to the broad Maxwellian distribution before expansion. The He atoms emerge from the nozzle with a broad angular distribution, and a skimmer is used in order to remove atoms moving not in the required direction. The beam is then directed towards the sample, which is placed in an ultra-high-vacuum chamber at a base pressure of better than $2 * 10^{-10}$ Torr, 
and mounted on a three axes manipulator that allows to vary and control the temperature of the sample continuously between $90 \mathrm{~K}$ and $1000 \mathrm{~K}$. Finally, the scattered He beam is detected by a differentially pumped quadrupole mass spectrometer.

The energy of the He beam can be continuously tuned simply by varying the temperature of the nozzle. With the nozzle held at room temperature, the He beam has an energy of about $65 \mathrm{meV}$, corresponding to a de Broglie wavelength of about $1 \AA$, which is on the order of interatomic distances in solids. Thus, just as in X-ray, electron or neutron scattering, the interaction of He atoms with the sample gives rise to diffraction phenomena, subject to the well known Bragg selection rules, which manifest itself through the fact that the angular distribution of scattered $\mathrm{He}$ atoms is sharply peaked under certain angles. This way, information on atomic scale properties of the surface or thin film can be deduced. Up- and downstream of the sample the He beam is collimated by series of narrow slits providing an angular resolution of about $0.2^{\circ}$. The momentum magnitude and direction of each $\mathrm{He}$ atom in the beam are thus sufficiently well defined that it can be described by a plane wave, which is coherent over a couple of hundred angstroms. Consequently, it is this extension on the surface, over which each individual $\mathrm{He}$ atom bears structural information through coherent interference of scattering events.

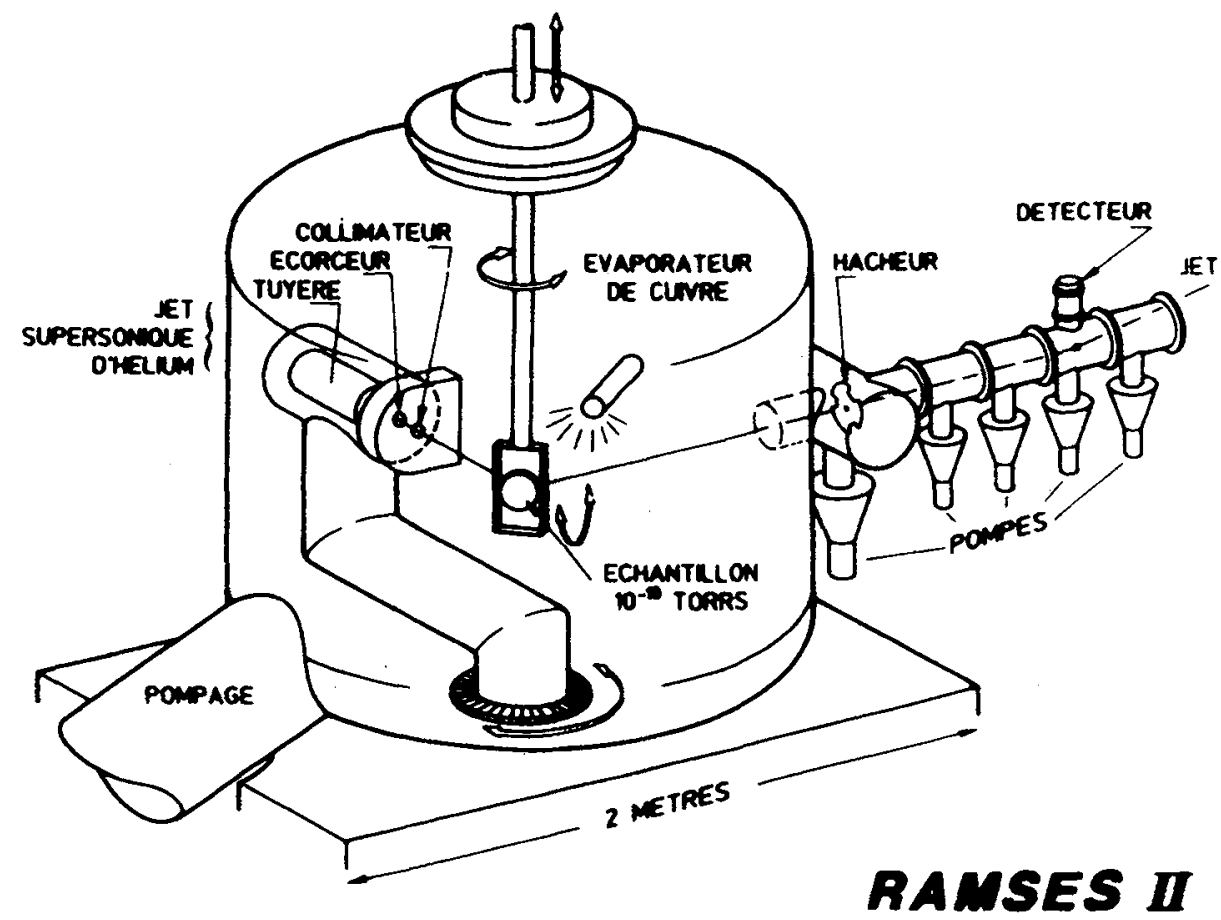

Figure 1: Sketch of the HAS spectrometer "Ramses II" at Saclay (see text for details)

As mentioned above, Helium scattering is in may ways analogous to X-ray, electron or neutron scattering, however, there is also a major difference. He atoms are strongly repelled as they enter the region of weak electronic charge density above the solid (the classical turning point is about $3 \AA$ above the ion cores of the outermost layer [3]). In other words, He atoms do not penetrate into the solid, which makes this probe extremely surface sensitive. Moreover, HAS is a non-destructive surface diffraction technique, a characteristic that will most likely prove to be very valuable in future studies of fragile organic 
materials. Finally, we mention an at first sight trivial property of HAS, which, however, from a practical point of view turns out to be particularly useful for the investigation of growth and ablation: this technique uses non-charged probe particles. It is therefore relatively straightforward to investigate such processes in 'real time', i.e. to perform the actual experiment during growth or ablation, free from the distorting influence of electrical or magnetic fields of evaporation or sputtering sources, from which techniques using charged probe particles often suffer. This 'real time' feature is certainly also still the most prominent advantage of HAS with respect to Scanning Tunneling Microscopy (STM), the inherent benefit of a real space technique covering a wide range in spatial resolution, including atomic, not withstanding.

In contrast to most existing helium atom beam spectrometers, Ramses II allows to rotate both the beam source and the sample continuously over a wide range of angles. This way, different perpendicular momentum transfers can be accessed rapidly, without the need to change the beam energy. This option is particularly useful for the investigation of atomically rough surfaces. Details concerning the evaporation source and the preparation of the $\mathrm{Cu}$ samples can be found elsewhere [4]

\section{RESULTS}

\subsection{Submonolayer morphology}

\subsubsection{The migration coefficient}

The mobility of deposited atoms is a decisive parameter for the quality of the grown film. Unfortunately, reliable numbers for activation energies are generally not available. Values for bulk diffusion are not very useful in thin film growth, even as a starting point, because the mobility in the bulk can vary orders of magnitudes with respect to diffusion on surfaces of the same material. Field Ion Microscopy (FIM) has provided most of our insights of how diffusion on surfaces occurs, however, this technique is restricted to a very limited number of materials due to the high electrical field used for imaging.
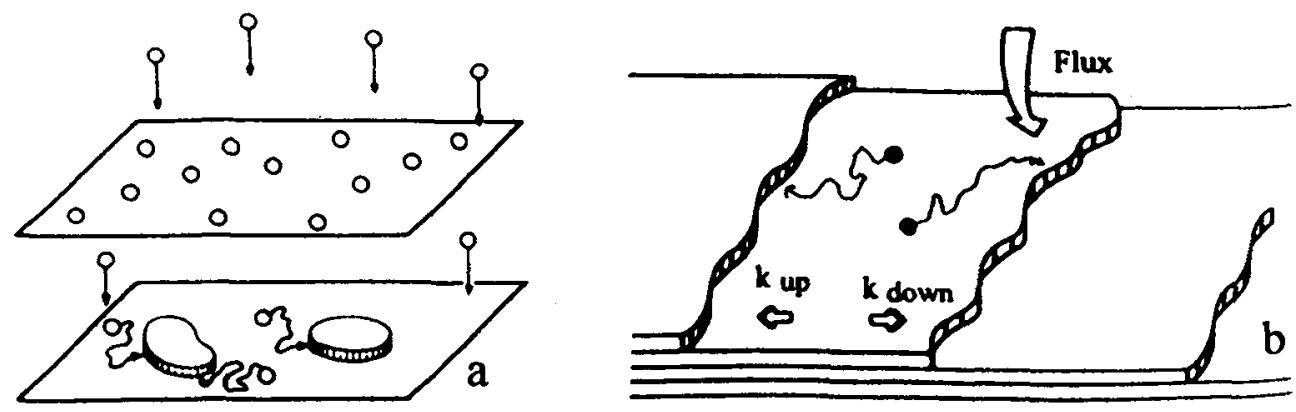

Figure 2: Growth mechanisms a) on flat (singular) surfaces, growth proceeds by nucleation and island formation b) on stepped (vicinal) surfaces, growth takes place via step flow (adapted from Ref. 16 and 28). The stability of the advancing step train depends on the adatom attachment coefficients $k$ to ascending and descending steps.

An other way to determine the migration coefficient is based on the following idea. The deposition of adatoms drives the system out of equilibrium, since the number of defects (adatoms) created this way does not correspond to the equilibrium concentration at that temperature. The system tries to restore equilibrium; this is done by forming islands (cf. Fig. 1a). Due to encounters of mobile adatoms, stable nuclei are formed which subsequently grow by incorporation of additional adatoms. Typically, the mean separation between islands is not random, because adatoms landing in the vicinity of already existing islands are more likely to be incorporated instead of forming a new nucleus. Thus, the spatial island distribution is expected to be correlated. This correlation should give rise to characteristic diffraction peaks 
in the angular distributions, which have in fact been identified in our HAS experiments [5], and subsequently as well with other surface sensitive diffraction techniques [6].

This close relation between the migration coefficient and the island density has been explored for many years in the past, mostly with scanning electron microscopy, however, in general only effective values for migration coefficients are reported, because in these experiments due to limited spatial resolution diffusion distances are typically of the order of micrometers: the use of atomic resolution techniques in order to determine the mean island separation has the merit that growth parameters can be chosen so that islands nucleate in their majority between preexisting defects and their influence on the migration of adatoms can be largely neglected.

Intuitively, one expects that the mean separation between islands depends essentially on the arrival rate of adatoms, the size of the critical nucleus, and the mobility of adatoms. A detailed quantitative description has been developped on the basis of rate equations [7]. In order to determine the migration coefficient, the mean separation between islands has to be measured as a function of temperature. However, if the critical nucleus size is not one (i.e. if the smallest stable island is not a dimer), the activation energy deduced from an Arrhenius plot contains more than just the migration activation energy, but as well atomic binding energies of islands [7]. Thus, for a reliable quantitative analysis, the size of the critical nucleus has to be known. This information is available from measurement of the mean island separation as a function of incident adatom arrival rate $[5,6]$. It appears imperative to determine the flux dependence very accurately over a wide range of temperatures, because the size of the critical nucleus itself can depend strongly on temperature. In fact, the safest way to determine the adatom migration activation energy is to identify the temperature range, in which the dimer is the stable nucleus. In principle, this is expected at only low enough substrate temperatures (unless the dimer formation is prohibited due to constraints imposed by the underlying lattice structure). In this case, the number of active kinetic processes is reduced to a minimum, and the energy deduced from an Arrhenius graph is directly proportional to the activation energy for adatom migration. Of course, this approach does not allow for an identification of the actual diffusion mechanism, such as for example simple hopping or more complicated concerted motions. For this purpose, one has to rely on comparison with calculations

The analysis of our HAS data along these lines for the deposition of $\mathrm{Cu}$ on $\mathrm{Cu}(001)$ gives an activation energy of $0.28 \mathrm{eV}$ for the migration of a $\mathrm{Cu}$ atom on this surface [5], under the assumption that the $\mathrm{Cu}$ dimer forms the stable nucleus in the whole temperature range covered by our experiment. Our flux dependent measurements of the mean island separation for different substrate temperatures have given already hints that this assumption should have been relaxed, and there is solid experimental evidence by now [6] that this system is characterized by a change in critical nucleus size from monomer to trimer at a surface temperature of about $220 \mathrm{~K}$. Then, the evaluation gives $0.36 \mathrm{eV}$ for the migration activation energy, and an average binding energy per bond of about $0.06 \mathrm{eV}$ [6]. Curiously, these values are significantly at variance with calculations based on the embedded atom method [8] or the corrected effective medium theory [9], which reveal both much larger binding energies and migration activation energies of only about $0.2 \mathrm{eV}$, favoring an exchange diffusion mechanism in this system. A comprehensive discussion of these unresolved discrepancies can be found in reference [6]. A first principles treatment of the diffusion process would be highly desirable.

\subsection{2 "Spinodal Decomposition" in 2D}

The concept of a "dynamical scaling law" in ordering phenomena seems to be generic [10]. Indeed, often the structural evolution can be described in terms of a single characteristic length that increases with time according to a simple algebraic law, whose associated exponent depends merely on very general properties of the system under investigation, such as its symmetry or its dimensionality. Moreover, often the ordering pattern is found to evolve in self similar fashion: at a given time the pattern has an appearance similar to an appropriately magnified portion of it at an earlier time.

Lifshitz and Slyozov [11] examined a long time ago a two component system undergoing phase separation with widely spaced domains of one phase growing in the matix of the second phase. Under these conditions, ordering is mediated by evaporation of particles from small domains, diffusion through the matrix, and subsequent re-condensation onto large domains. In that case, the theory reveals that the power law exponent amounts to $1 / 3$, and many experiments have confirmed this prediction. It is a priori 
not clear if this result still holds, when the volume fractions of the two phases are comparable, as it is for example realized in spinodal decomposition of an alloy. Here, the morphology is charaterized by an interconnected and interpenetrating domain network instead of isolated domains, and it is conceivable that this particular structure opens the possibility for different ordering mechanisms.

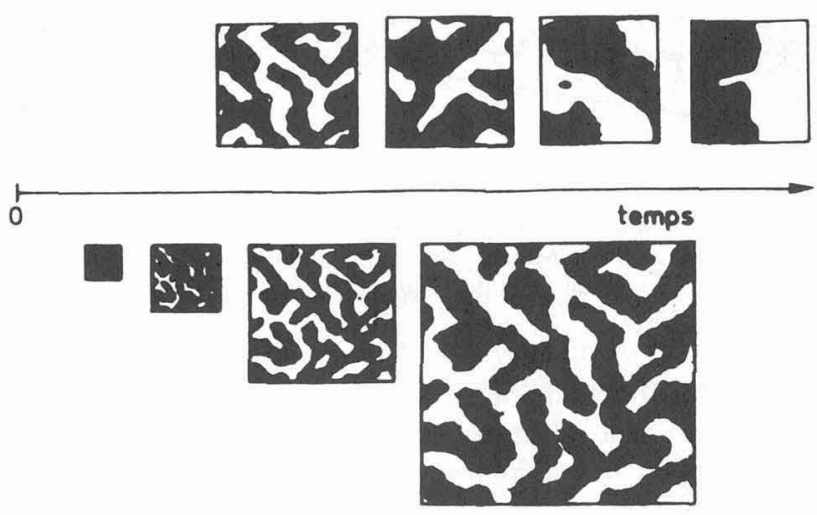

Figure 3: Sketch of an interconnected domain network formed by depositing half a monolayer $\mathrm{Cu}$ on a $\mathrm{Cu}(001)$ surface, and its selfsimilar temporal evolution: at a given time the pattern has an appearance similar to an appropriately magnified portion of it at an earlier time (adapted from Ref. 10). In the time and temperature range covered by our experiment, ordering proceeds by mass transport along domain boundaries only.

We have addressed this question for a two dimensional system using HAS as a probe. However, instead of using a real alloy, whose surface properties are difficult to control due to segregation and diffusion of particles onto the surface and into the bulk, we have produced a $2 \mathrm{D}$ system with equal volume fractions of the two phases by depositing 0.5 monolayer $\mathrm{Cu}$ on a $\mathrm{Cu}(001)$ surface at $100 \mathrm{~K}$. At this temperature, adatom diffusion is largely negligible and a network of interconnected and interpenetrating domains forms. The system is subsequently rapidly raised to and held at a higher temperature, and the kinetics of domain growth is monitored by measuring diffraction profiles as time evolves $[12,13]$. We find that the morhology evolves indeed in a self-similar fashion, and an exponent close to $1 / 4$ to the algebraic law. This value is in accord with ideas of corrections [14] to the Lifshitz-Slyozov growth law and implies that ordering proceeds by mass transport along domain boundaries only in the temperature and time range covered by our experiment.

\subsection{Multilayer Morphology}

\subsubsection{Growth instability on singular surfaces}

Akin to the scaling properties in ordering phenomena, the growth of crystals is believed to obey as well dynamical scaling laws [15]. However, in contrast to ordering processes, for which the rescaling is isotropic and uniform in every spatial direction, the growth front in deposition processes is believed to evolve in a self-affine fashion, i.e. a different rescaling of characteristic lengthes parallel and perpendicular to the growing film is needed in order to identify patterns in the statistical sense for different deposition times (coverage). Here again, both characteristic lengthes are supposed to evolve with time according to simple algebraic laws. The associated exponents are distinctive for a specific class of growth models and thus allow to identify the dominating underlying ordering mechanism [15]. Recently, a different growth model has been introduced by $J$. Villain [16]. He points out that the growth front may not evolve in a selfaffine manner, but instead in the form of pyramidlike surface profiles, if the adatom's ability to move from one atomic layer to another is kinetically limited. This asymmetry in the horizontal and vertical diffusion is due to the presence of an excess energy barrier associated with steps (the so called Ehrlich-Schwöbel barrier $[17,18])$. 

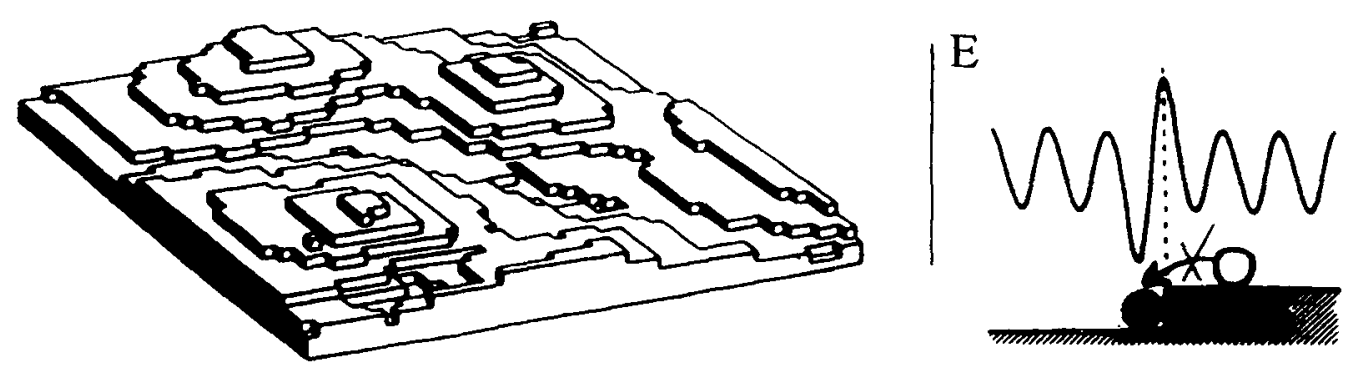

Figure 4: "Les pyramides de Ramses II". Multilayer-deposition of $\mathrm{Cu}$ on $\mathrm{Cu}(00 \mathrm{l})$ at $200 \mathrm{~K}$ leads to the formation of pyramidlike patterns, similar to those obtained in a Monte Carlo simulation by $P$. Smilauer et al. (Ref. 21), which incorporates an aysmmetry in the horizontal and vertical mobility of adatoms due to an excess energy barrier at steps.

Our HAS experiments reveal the formation of such a pyramidlike growth front in the deposition of about 100 monolayers $\mathrm{Cu}$ on $\mathrm{Cu}(001)$ at a substrate temperature of $200 \mathrm{~K}[19,20]$. It is likely that the origin of this patterned structure is indeed the asymmetry in the adatom's intra- and interlayer mobility. Further support comes from the observation [4] that the spatial separation of these pyramidlike structures corresponds roughly to the island separation found at the very early stages of growth: it is then when steps bordering large islands are formed, and the excess energy barrier can restrain interlayer mass transport. Our HAS experiments during deposition indicate that the angle between the base and sides of the pyramids increases slowly with deposition time, and converges finally to a limiting value of precisely $15.8^{\circ}$. This is the angle between the terrace and the macroscopic orientation found in a $\mathrm{Cu}(115)$ surface. Monte Carlo simulations by Amar and Family [22] and Bartelt and Evans [23] of the growth process, which correctly include the fcc geometry of the surface, show that the specific angle selection results from the competition between an adatom 'downhill current' [23] produced by the requirement that overhangs do not form and adatoms are placed in fourfold hollow sides only, and an 'uphill current ${ }^{1}{ }^{2}$ [24] due to the Ehrlich-Schwöbel barrier. Very recent electron diffraction experiments [25] reveal that this slope selection, in combination with a laterally anisotropic advancement rate of island edges imposed by the fcc geometry, can lead even to the formation of a surprisingly regular, checkerboardlike arrangement of the pyramidal structures in a certain coverage range. Marvelous real space views of these "pyramides de Ramses II" by means of STM are meanwhile available [40].

\subsubsection{Growth instabilities on vicinal surfaces}

Realizing the decisive influence of steps for the mobility of deposited atoms, we have studied growth on so called vicinal surfaces. These are surfaces with orientations tilted over a small angle with respect to a high symmetry (singular) direction, thus they consist at the outset of a regular arrangement of steps separated by terraces. In material science, growth on such templates has been recognized as an alternative, promising route to tailor atomically abrupt and smooth interfaces. In the so called 'step flow growth mode', deposited atoms attach directly without island formation to preexisting steps (see Fig. 1b). This advances the steps perpendicular to themselves, and, in steady state, a step train flows across the surface and growth proceeds in a layer by layer fashion.

\footnotetext{
'The term 'uphill current' does not mean that the adatoms 'jump up the step'. They are captured by the ascending step; however, they exhibit a greater probability of moving towards the ascending step than towards the descending.
} 
This mechanism respects thus the self-replication property that is essential for 'good growth', provided that the flowing step train is stable. However, often, step bunching and faceting has been observed, an undesirable effect for most applications. Now, there are many sources for a destabilization of the step train, such as impurities acting as pinning centers, or steric constraints due to surface reconstructions, but also intrinsic origins related to the adatom attachment kinetics to ascending and descending steps [26]. In order to address the latter aspect, we have investigated the growth via step flow in homoepitaxial, nonreconstructed systems under ultra high vacuum conditions.

Burton, Cabrera and Franck [27] have studied step flow growth a long time ago under the assumption that adatoms bond to ascending and descending steps with equal rates. However, in the presence of the excess energy barrier to migration over descending steps, an adatom 'uphill current' is produced, and therefore, the capture rate coefficients to steps become directionally dependent. Consequently, a terrace, which for example is larger than its upper neighbor, receives more adatoms from the incoming flux which then attach preferentially to the ascending step; this increases the velocity of the upper bounding step and as a result the average terrace width is restored [26]. This 'negative feedback' argument would imply that step flow should be stable in the presence of a Schwoebel-Ehrlich barrier However, as pointed out first by Bales and Zangwill [28], the same situation can provoque a growth instability along step edges, which manifests itself by a transverse meandering of steps.

Our HAS experiments [29] on the growth of $\mathrm{Cu}$ on vicinal $\mathrm{Cu}$ surfaces provide evidence for the existence of the Bales-Zangwill instability. We find that homoepitaxial step flow on $\mathrm{Cu}(1,1,17)$ below room temperature produces a collective, in phase meandering of steps and thus facets parallel to the average step direction, while the average terrace width perpendicular to the average step direction is maintained. Step flow on $\mathrm{Cu}(1,1,5)$ in this temperature range leads as well to a meandering of $(1,1,5)$ steps, without, however, a specific phase selection. In contrast to the $\mathrm{Cu}(1,1,17)$, growth on $\mathrm{Cu}(1,1,5)$ above room temperature produces a faceting of the surface in the direction perpendicular to the average step direction [29]. In terms of the inherent kinetics of the step train, the latter observation asserts [26] that on this surface the uphill adatom current present at low temperatures, which guarantees the stability of the train with respect to the average terrace distance between steps, must be overcompensated by a downhill current at high enough temperatures. The exact origin of the downhill current is not yet setteld. We may discuss conceivable origins.

The strength of the uphill current in step flow growth is proportional to the width of the terraces separating steps [27], and the stability of the step train with respect to the average terrace width is governed by the difference in downhill and uphill current $[26,28]$. Because only the $(1,1,5)$ surface, which has an about three times smaller terrace width than the $(1,1,17)$ surface, is observed to be destabilized this way in the temperature range covered by our experiment, one may anticipate that the change in sign of the net surface current results from the activation of specific kinetic processes in the immediate vicinity of descending steps only. This suggests that for example exchange mechanisms, characterized not only by a higher energy barrier, but also a higher pre-exponential factor [30], or 'knock out' effects [31] at the descending step edge become dominant with temperature. In this respect, the $\mathrm{Cu}(1,1,5)$ surface, due to its short terraces, could then be a marginal case. On the other hand, the small step separation on this surface may imply that in this case a reasoning exclusively in terms of the adatom attachment kinetics fails, and that step-step interactions could play an essential role in this faceting process. In fact, there is growing evidence that step interactions are more complicated than previously thought; they could even be attractive for small step separations [32,33]. The delicate balance of forces that otherwise guaranties the stability of the surface under equilibrium conditions (at least for temperatures as high as $680 \mathrm{~K}$ [34]) could then be distorted by the presence of the adatom diffusion field and finally lead to a faceting of the surface [35] Clearly, this interesting phenomenon needs further investigation.

\subsection{Ablation}

Besides its technological importance for example in ion beam machining, ion beam assisted deposition or depth profiling, ablation may give complementary information as to the thin film growth processes described above. Here again, HAS turns out to be a very useful technique due to its possibility to observe the structural evolution even in the presence of the Ar ion beam used to remove material. 
In the investigation of growth processes with diffraction techniques, the temporal evolution of diffraction peak intensities is often used as a 'fingerprint' in order to judge the quality of the growing crystal. In the anti-phase condition [36], the diffracted intensity depends on the difference between uncovered odd and even numbered layers. Consequently, when the film grows in a layer by layer fashion, oscillations in intensity with deposition time are observed. By contrast, the absence and rapid damping of the diffracted intensity is taken as a signature of three-dimensional growth.

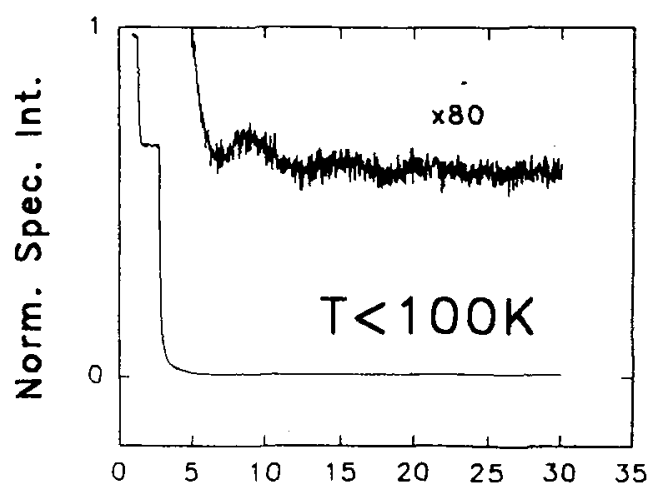

\section{Bombardment time [100 sec]}

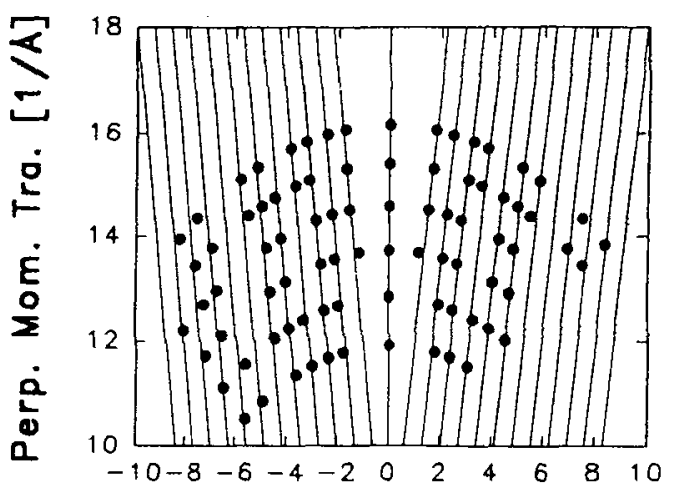

Parallel Moment. Transf. [1/A]

Figure 5: Specularly diffracted He intensity during bombardment of a $\mathrm{Cu}(001)$ surface with $350 \mathrm{eV} \mathrm{Ar}$ ions (flux $\approx 2 \times 10^{12}$ ions $/ \mathrm{cm}^{2} \mathrm{sec}$, incident angle $\approx 30^{\circ}$ ) at a surface temperature lower than $100 \mathrm{~K}$ (left). Weak oscillations are seen, indicative of a certain degree of layer by layer removal (the initial drop in intensity is due to He-Ar gas phase scattering). Ion bombardment at $325 \mathrm{~K}$ leads to a HAS diffraction pattern (right), which reflects the formation of pyramidlike void structures at that temperature. The sides of the vacancy pyramids exhibit the geometrical structure of a $\mathrm{Cu}(1,1,7)$ surface, evidenced by the inclination angle and the periodicity of the lattice rods.

Fig. 5 shows the specularly diffracted intensity from a $\mathrm{Cu}(001)$ surface during bombardment with Ar ions at a substrate temperature below $100 \mathrm{~K}$. The signal shows some weak oscillations during ablation, indicating a cenain degree of layer by layer removal. By contrast, when the substrate is kept at higher temperatures during bombardment, no oscillations in the reflected intensity are observed, but instead, a monotonic decrease of the signal with bombardment time. An analysis of the entire HAS diffraction pattern reveals that at that temperature pyramidlike void structures are formed during ablation, whose sides exhibit the geometrical characteristics of a $\mathrm{Cu}(1,1,7)$ surface in this case [37]. Similar void structures have also recently been identified in STM investigations of ion ablation on $\mathrm{Cu}(001)[38,39]$.

In fact, our HAS experiments on the ablation with Ar ions covering a wide range of substrate temperatures reveal that, roughly speaking, the morphologies produced during growth of $\mathrm{Cu}$ on $\mathrm{Cu}(001)$ and removal of $\mathrm{Cu}$ from $\mathrm{Cu}(001)$ are 'reciprocal' to a large extent, subject to differences in the temperature range, for which adatom- and vacancy pyramidlike structures are formed. This suggests that the formation of these patterned structures have a common origin: the restriction of interlayer adatom and interlayer vacancy transport, respectively. Of course, in both cases the actual 'moving species' are atoms, which, in MBE are supplied directly, while in ablation they have to be created by detachment from step edges. The activation energies involved are certainly different, which thus explains that adatom and vacancy pyramids are formed in different temperature ranges during growth and ablation, respectively.

\section{CONCLUSION AND OUTLOOK}

HAS allows for a detailed characterization of surface and thin film morphologies in situ, on an angstrom scale, and in real time during both growth and ablation. The investigation of homoepitaxial systems has revealed the formation of a variety of selforganized patterns, which, in both growth and ablation, seem to 
have their common origin in an asymmetry in the vertical and horizontal mobility of atoms due to an excess energy barrier at step edges. These results emphasize the decisive role of kinetics in the growth and ablation of thin films. In fact, this asymmetry should exist in multicomponent (heteroepitaxial) systems as well, and represents therefore one of the fundamental aspects of thin film growth and ablation.

It is tempting to speculate, whether the step edge barrier can be modified by the presence of external, electric fields for example. This would offer the possibility to manipulate the growth or ablation mode in an easily controlable manner. On the other hand, the observed growth and ablation instabilites are not necessarily a nuissance; they may, in fact, represent a promising avenue for a lateral patterning of surfaces and interfaces on a nanometerscale, which is still one of the major challenges.

The HAS results concerning the ablation of surfaces may in fact open interesting perpectives. For example, a comparing real time observation of ion induced and Laser induced ablation could give detailed insights into the basic mechanisms involved in these atom removal processes. Also, the growth of metal insulator interfaces, the growth of thin organic films on metals - wide and technologically important fields, for which little atomic scale structural information is available. HAS seems to be particularly well adapted for a detailed investigation of such systems, which asks for a sensitive, non-charged and non-destructive probe particle. Certainly, "HAS is not a pursuit for the faint-hearted" (R.B. Doak in [3]), but once mastered, it is one of the best techniques a surface scientist can wish for.

\section{Acknowledgements}

I like to acknowledge the valuable contributions of F. Fabre, R. Folkerts, J. Lapujoulade and L. Schwenger at various stages of this project, and the expert technical assistance of P. Lavie, M. Lefort, and F. Merlet. I thank L. Schwenger as well for a critical reading of the manuscript. I wish to dedicate this work to the memory of Monsieur Michel Lefort, en hommage to his outstanding professional performance, and his exceptionnel humane qualities.

\section{References}

[1] L.J. Gomez et al., Phys. Rev. B 31, (1985) 255

[2] R. Kunkel et al., Phys. Rev. Lett. 65 (1990) 733

[3] "Helium Atom Scattering from Surfaces", ed. by E. Hulpke, Springer Series in Surf. Sci. 27, Springer Verlag 1992

[4] H.-J. Ernst et al., J. Vac. Sci. Techn. A12 (4) (1994) 1809

[5] H.-J. Ernst, F. Fabre, J. Lapujoulade, Phys. Rev. B 46 Rap. Comm. (1992) 1929

[6] J.-K. Zuo et al., Phys. Rev. Lett. 72 (1994) 3064; H. Dürr, J.F. Wendelken, J.-K. Zuo, Sur. Sci. Lett. 328 (1995) LS27

[7] J.A. Venables, G.D.T. Spiller, M. Hanbücken, Rep. Progr. Phys. 47 (1984) 399

[8] L.S. Perkins, A.E. DePristo, Surf. Sci. 325 (1995) 169

[9] L.B. Hansen et al., Surf. Sci. 289 (1993) 68

[10] H. Furukawa, Adv. Phys. 34 (1985) 704

[11] I.M. Lifshitz, V.V. Slyosov, J, Phys. Chem. Solids 19 (1961) 35

[12] H.-J. Ernst, F. Fabre, J. Lapujoulade, Phys. Rev. Lett. 69 (1992) 458

[13] H.-J. Ernst, F. Fabre, F. Lapujoulade, La Recherche 250 (1993) 90

[14] D. A. Huse, Phys. Rev. B 34 (1986) 7845

[15] A.-L. Barabasi, H.E. Stanley, "Fractal concepts in surface growth", Cambridge University Press 1995

[16] J. Villain, J. Phys. I 1 (1991) 19

[17] G. Ehrlich, F. Hudda, J. Chem. Phys. 44 (1966) 1039

[18] R.L. Schwoebel, J. Appl. Phys. 40 (1969) 614

[19] H.-J. Ernst et al., Phys. Rev. Lett. 72 (1994) 112

[20] Pour la Science 195 (1994) 18

[21] P. Smilauer, M.R. Wilby, D. Vvedensky, Phys. Rev. B 47 (1993) 4119 
[22] J. Amar, F. Family, Phys. Rev. B 54 (1996) 14742

[23] M.C. Bartelt, J.W. Evans, Phys. Rev. Lett. 75 (1995) 4250

[24] M. Siegert, M. Plischke, Phys. Rev. Lett. 68 (1992) 2035

[25] L.C. Jorritsma et al., Phys. Rev. Lett. 78 (1997) 911

[26] R.L. Schwoebel, E.J. Shipsey, J. Appl. Phys. 37 (1966) 3682

[27] W.K. Burton, N. Cabrera, F.C. Frank, Phil. Trans. R. Soc. Lond. 243 (1951)

[28] G.S. Bales, A. Zangwill, Phys. Rev. B 41 (1990) 5500

[29] L. Schwenger, R. Folkerts, H.-J. Ernst, Phys. Rev. B 55, Rap. Comm. (1997) R7406

[30] G. Boisvert, L. Lewis, A. Yelon, Phys. Rev. Lett. 75 (1995) 469

[31].D.D. Vvedensky et al., Phys. Rev. E 48 (1993) 852

[32] M.S. Papadia, M.C. Desjonquères, D. Spanjaard, Phys. Rev. B 53 (1996) 4083

[33] J. Frohn et al., Phys. Rev. Lett. 67 (1991) 3543

[34] H.-J. Ernst, R. Folkerts, L. Schwenger, Phys. Rev. B 52 (1995) 8461

[35] M. Lässig, Phys. Rev. Lett. 77 (1996) 526

[36].H.-J. Ernst, F. Fabre, J. Lapujoulade, Surf. Sci. 275 (1992) L682

[37] H.-J. Ernst, accepted for publication in Surf. Sci. Lett.

[38] M. Ritter et al., Surf. Sci. 348 (1996) 243

[39] J.C. Girard et al., Surf. Sci. 302 (1994) 73

[40] J.-K. Zuo, J.F. Wendelken, Phys. Rev. Lett. 78 (1997) 2791 